\title{
Linfoma hepático primario. Evolución favorable con quimioterapia combinada con rituximab
}

\author{
I. Serrano-Navarro, J. F. Rodríguez-López, R. Navas-Espejoํㅜ, M. A. Pérez-Jacoiste, \\ M. A. Martínez-González ${ }^{1}$, C. Grande ${ }^{2}$ y S. Prieto \\ Servicios de Medicina Interna, ${ }^{1}$ Anatomía Patológica y ${ }^{2}$ Hematología. Hospital Universitario 12 de Octubre. Madrid
}

\begin{abstract}
RESUMEN
Comunicamos el caso de una paciente con un linfoma hepático primario tratado con éxito con quimioterapia combinada con rituximab. Utilizando los "encabezamientos estándar para búsquedas bibliográficas informatizadas" (Medical Subject Heading) revisamos los casos publicados hasta la fecha de esta infrecuente entidad.
\end{abstract}

Palabras clave: Linfoma primitivo de hígado. Linfomas no hodgkinianos. Rituximab.

\begin{abstract}
This article describes the case of a patient with a non-Hodgkin primary hepatic lymphoma who was successfully treated with chemotherapy combined with rituximab. Using the Medical Subject Headings the published reports of this rare entity were reviewed.
\end{abstract}

Key words: Primary hepatic lymphoma. Non-Hodgkin's lymphomas. Rituximab.

Serrano-Navarro I, Rodríguez-López JF, Navas-Espejo R, Pérez-Jacoiste MA, Martínez-González MA, Grande C, Prieto S. Linfoma hepático primario. Evolución favorable con quimioterapia combinada con rituximab. Rev Esp Enferm Dig 2008; 100: 724-728.

\section{INTRODUCCIÓN}

El linfoma de localización primariamente hepática es una entidad excepcional con manifestaciones clínicas inespecíficas, que debe figurar en el diagnóstico diferencial de las lesiones ocupantes de espacio en ese parénquima. Partiendo de la revisión de Noronha y cols. publicada en 2005 (1), en la que describían 251 casos comunicados hasta febrero de 2003, realizamos una búsqueda en Medline de los casos de las entidades "lymphoma" y "liver neoplasms" publicados hasta julio de 2007. Por su rareza y posible interés, revisamos las características de esta entidad en función del caso de una paciente ingresada por afectación del estado general y sugerencia de metástasis hepáticas en la tomografía computadorizada.

Recibido: $18-03-08$.

Aceptado: 03-06-08.

Correspondencia: S. Prieto. Servicio de Medicina Interna. Hospital 12 de Octubre. Avenida de Córdoba, s/n. 28041 Madrid. e-mail: sprieto.hdoc@salud.madrid.org

\section{CASO CLÍNICO}

Mujer de 68 años sin hábitos tóxicos ni antecedentes de interés, que ingresa por un cuadro de dolor continuo en hipocondrio derecho, astenia, anorexia y pérdida de cinco quilos de peso desde un mes antes. En las semanas previas no había observado fiebre, síntomas gripales, respiratorios ni digestivos.

\section{Examen físico}

Afectación del estado general; eupneica; normal coloración cutaneomucosa; no se palpaban tiroides ni adenomegalias laterocervicales, supraclaviculares $\mathrm{o}$ axilares. TA: $130 / 70 \mathrm{mmHg}$; temperatura $37,0^{\circ} \mathrm{C}$. Auscultación cardiopulmonar: normal. Abdomen: hígado palpable a tres centímetros de reborde costal derecho y cinco centímetros de xifoides, superando la línea media, firme y doloroso; bazo no palpable. Extremidades: sin alteraciones. 


\section{Exámenes complementarios}

Hemograma: hemoglobina $(\mathrm{Hb}) 12 \mathrm{~g} / \mathrm{dl}$ (normal 1216); Hto $36 \%$ (36-48); VCM 86 fl (80-100); leucocitos $7.800 / \mathrm{mm}^{3}$ (4.500-10.000); plaquetas $228.000 / \mathrm{mm}^{3}$ (140.000-400.000). Actividad de protrombina 97\% (70120); tiempo de cefalina 27 segundos (25-34 s); fibrinógeno $506 \mathrm{mg} / \mathrm{dl}$ (200-400). Glucemia $103 \mathrm{mg} / \mathrm{dl} \mathrm{(70-}$ 105); creatinina $0,79 \mathrm{mg} / \mathrm{dl}(0,7-1,10)$; GOT $115 \mathrm{U} / \mathrm{l}$ (5-45); GPT 126 U/l (5-45); LDH 860 U/1 (90-230); GGT $111 \mathrm{U} / \mathrm{l}(3-52)$; bilirrubina $0,98(0,2-1,0) \mathrm{mg} / \mathrm{dl}$; fosfatasa alcalina $393 \mathrm{U} / 1$ (98-295); proteínas totales 6,2 $\mathrm{g} / \mathrm{dl}(6,3-8,0)$, con $3,46 \mathrm{~g} / \mathrm{dl}$ de albúmina $(3,2-5,5)$ y espectro electroforético normal; calcio $9,6 \mathrm{mg} / \mathrm{dl}(8,4-$ $10,2)$; fósforo $3,4 \mathrm{mg} / \mathrm{dl}(2,3-4,6)$; ácido úrico $5 \mathrm{mg} / \mathrm{dl}$ (2,2-7,0); colesterol $159 \mathrm{mg} / \mathrm{dl}$ (150-200); triglicéridos $65 \mathrm{mg} / \mathrm{dl}$ (50-170); sodio $141 \mathrm{mEq} / \mathrm{l}$ (136-145); potasio $4,3 \mathrm{mEq} / \mathrm{l}(3,5-5,2)$; sideremia $45 \mu \mathrm{g} / \mathrm{dl}(60-120)$; ferritina $282 \mathrm{ng} / \mathrm{ml}(30-400)$, transferrina $185 \mathrm{ng} / \mathrm{dl}$ (200360). Marcadores tumorales: antígeno CA 15,3: 12 $\mathrm{UI} / \mathrm{ml}$ (normal < 30), CA 54,9: $3 \mathrm{UI} / \mathrm{ml}(<12)$, CA 125: $5 \mathrm{UI} / \mathrm{ml}(<35)$, CA 19,9: $8 \mathrm{U} / \mathrm{ml}(<37)$, antígeno carcinoembrionario: $3 \mathrm{U} / \mathrm{ml}(<5)$ y tripsina: $180 \mathrm{ng} / \mathrm{ml}(140-$ $400) ; \beta_{2}$ microglobulina: $4,2 \mathrm{mg} / 1$ (1,2-2,8 mg/l). Serologías de virus B y $\mathrm{C}$ de hepatitis y VIH: negativas. Electrocardiograma: sin alteraciones. Radiografía de tórax: sin alteraciones. Radiografía simple de abdomen: hepatomegalia. Ecografía abdominal: múltiples lesiones sólidas en hígado, de uno a tres centímetros de diámetro, sugerentes de metástasis; sin otros hallazgos. TC toracoabdominal: múltiples lesiones, sólidas e hipodensas, ocupantes de la práctica totalidad del hígado, de diámetro similar al hallado en la ecografía; no se hallan adenomegalias axilares, retroperitoneales ni mesentéricas (Fig. 1). Colonoscopia: sin hallazgos. Punción-aspiración con aguja fina (PAAF) de una lesión hepática con control de TC: linfoma no hodgkiniano. Biopsia hepáti-

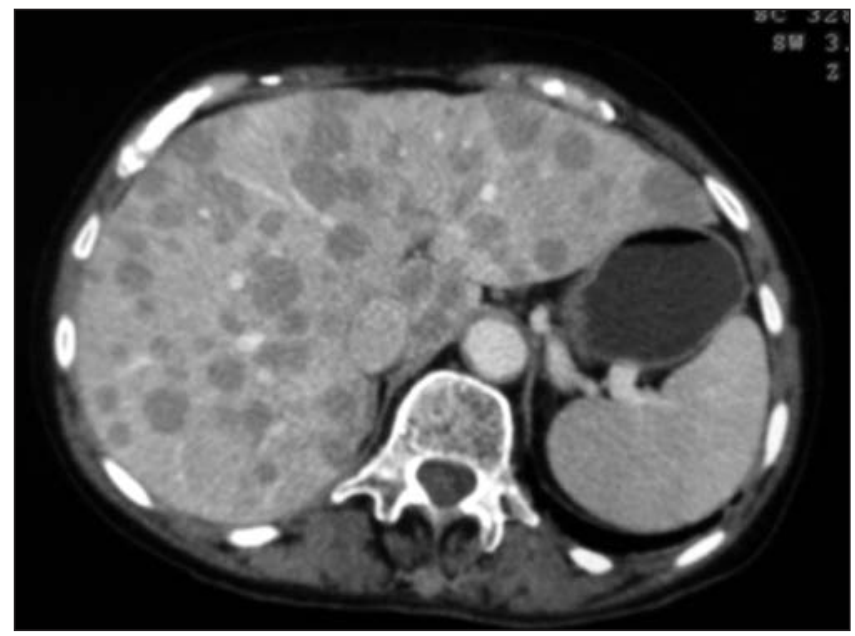

Fig. 1. TC abdominal en el momento del diagnóstico. Múltiples lesiones hepáticas, sólidas e hipodensas, de uno a tres centímetros de diámetro. ca con control de TC: linfoma B difuso de células grandes (Fig. 2). Inmunohistoquímica: células atípicas con fenotipo linfoide B (CD20 y CD79 $\alpha$ positivos), positivas para $\mathrm{CD} 10$ y bcl 6 , y negativas para marcadores $\mathrm{T}$ (CD3); CD 43, bcl 2 y queratinas AE1-AE3: negativas (Fig. 3). Mantoux: 9 mm. Estudio de médula ósea: aspirado sin hallazgos patológicos; coágulo y biopsia de médula ósea sin signos de infiltración. Biología molecular: región CDR1 del gen IgH policlonal. Estudio de líquido cefalorraquídeo: sin alteraciones bioquímicas ni citológicas. TC craneal normal. Gammagrafía con ${ }^{67} \mathrm{Ga}-$ lio: sin hallazgos patológicos.

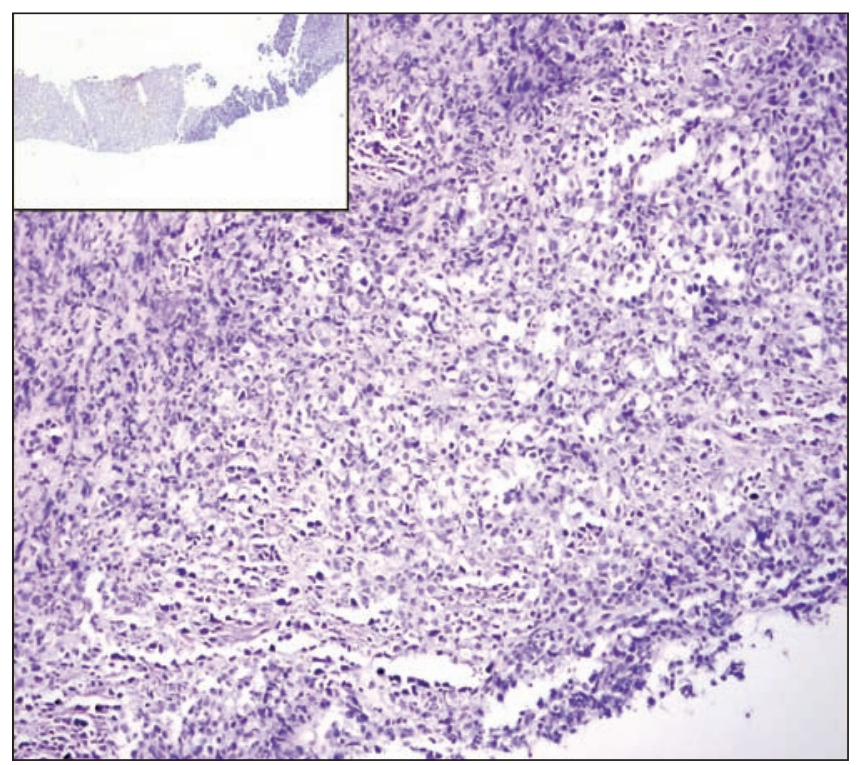

Fig. 2. Cilindro de tejido hepático que muestra el parénquima desplazado por una proliferación neoplásica de estirpe linfoide constituida por células de tamaño intermedio y grande, con núcleos irregulares y nucléolos prominentes.

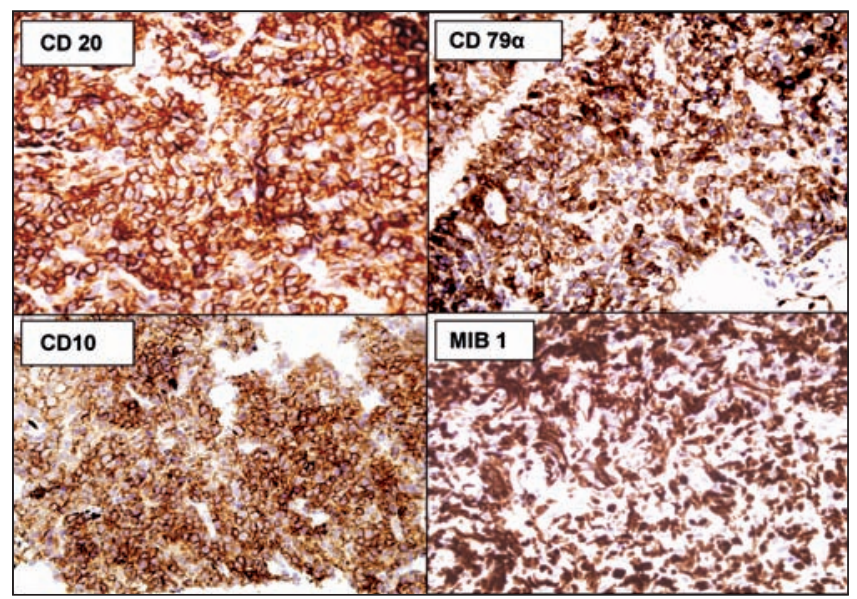

Fig. 3. Las células neoplásicas expresan CD20 y CD79 $\alpha$ (linfocitos de fenotipo B) y CD10. El índice proliferativo (MIB-1) es del $90 \%$. 


\section{Evolución}

Los datos bioquímicos empeoraron durante el periodo de estudio, alcanzando los siguientes valores: bilirrubina $2,8 \mathrm{mg} / \mathrm{dl}$, GOT $196 \mathrm{U} / \mathrm{l}$, GPT $109 \mathrm{U} / \mathrm{l}$, fosfatasa alcalina 878 U/1, GGT 911 U/1 y LDH 3.973 U/l.

La paciente fue tratada con fluidos parenterales, prednisona y dosis bajas diarias de ciclofosfamida a modo de prefase, además de quimioprofilaxis para la tuberculosis con isoniacida. A los seis días de tratamiento, y ya normalizadas las cifras de bilirrubina, recibió un primer ciclo de quimioterapia tipo R-CHOP (rituximab, $375 \mathrm{mg} / \mathrm{m}^{2}$; ciclofosfamida, $750 \mathrm{mg} / \mathrm{m}^{2}$; adriamicina $50 \mathrm{mg} / \mathrm{m}^{2}$; vincristina $1,4 \mathrm{mg} / \mathrm{m}^{2}$ y prednisona $100 \mathrm{mg} /$ día, cinco días), junto con quimioterapia intratecal triple y soporte con factor estimulante de progenitores granulocíticos. Toleró bien el tratamiento y al final del primer ciclo presentaba los siguientes datos bioquímicos: bilirrubina $1,3 \mathrm{mg} / \mathrm{dl}$; GGT 373 U/1; fosfatasa alcalina 361 U/l; LDH 397 U/1 y aminotransferasas dentro de la normalidad.

Debido al elevado índice pronóstico internacional (IPI) inicial, a las tres semanas recibió un segundo ciclo de quimioterapia, esta vez con R-MegaCHOP (rituximab $360 \mathrm{mg} / \mathrm{m}^{2}$; ciclofosfamida $1.500 \mathrm{mg} / \mathrm{m}^{2}$, adriamicina $65 \mathrm{mg} / \mathrm{m}^{2}$; vincristina $1,4 \mathrm{mg} / \mathrm{m}^{2}$, y prednisona $100 \mathrm{mg} /$ día, cinco días). En esta ocasión también se asoció terapia intratecal triple, bien tolerada.

Dada de alta, recibió posteriormente cuatro ciclos más de quimioterapia según protocolo R-MegaCHOP. Tras el tercero de ellos reingresó por un cuadro de neutropenia febril (leucocitos: $1.560 / \mathrm{mm}^{3}-1440 \mathrm{~N}, 90 \mathrm{~L}, 30 \mathrm{M}-$ ) y síntomas de infección de vías respiratorias altas, aislándose en el exudado nasal un virus respiratorio sincitial. Fue tratada con éxito con ribavirina inhalada y dada de alta al séptimo día con 2.490 leucocitos $/ \mathrm{mm}^{3}(1490 \mathrm{~N}, 460 \mathrm{~L}, 400 \mathrm{M}, 140 \mathrm{E})$.

Tras seis ciclos de quimioterapia las enzimas hepáticas y la $\beta_{2}$ microglobulina eran normales, y en la TC de control las lesiones eran inapreciables (Fig. 4). Dos años des-

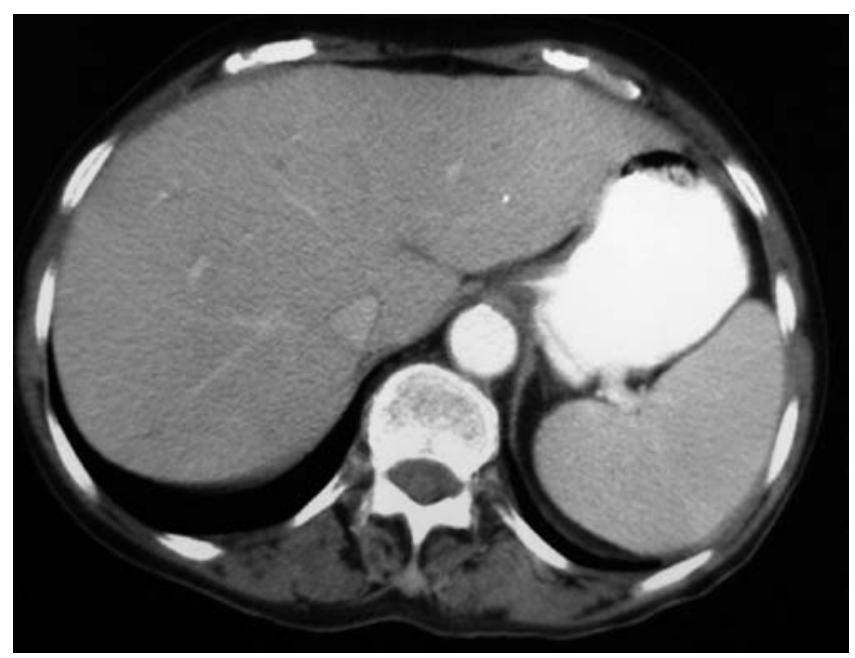

Fig. 4. TC abdominal tras seis ciclos de quimioterapia. Desaparición de las lesiones ocupantes de espacio en hígado. pués del diagnóstico la paciente se halla en remisión completa, mantiene buen estado general y sigue las revisiones programadas en consulta ambulatoria.

\section{DISCUSIÓN}

Las lesiones malignas halladas en hígado mediante técnicas de imagen constituyen una entidad no excepcional, ya que ese es, tras los ganglios linfáticos, el tejido afectado por metástasis con más frecuencia. En muchas ocasiones, la neoplasia primaria ya es conocida de antemano, o sospechada tras la anamnesis y el examen físico; en otras, una historia de cirrosis hepática o infección por virus $\mathrm{B}$ o $\mathrm{C}$ de hepatitis sugiere un carcinoma primitivo hepatocelular; finalmente, en otros casos, la citología obtenida por punción-aspiración con aguja fina, o la biopsia, orientan a la índole histológica y origen de la neoplasia y, sólo excepcionalmente, permiten el diagnóstico de un linfoma primitivo hepático $(2,3)$.

Comentamos el caso de una paciente sin antecedentes significativos que ingresó por un cuadro de afectación del estado general, hepatomegalia dolorosa, alteraciones enzimáticas y lesiones focales hepáticas en la TC, que citológica e histológicamente correspondían a un linfoma B difuso de células grandes.

Para que un linfoma pueda ser considerado como primitivamente hepático (LHP), en el momento del diagnóstico los síntomas han de obedecer a la afectación de ese parénquima y no haber sido demostrada la afectación de bazo, ganglios linfáticos, sangre periférica, médula ósea u otros tejidos hasta al menos seis meses después. Según esos estrictos criterios de Caccamo (4), Lei identificó 90 casos entre 1981 y 1993 (5), Noronha describió 251 publicados entre 1981 y 2003, y nosotros hemos hallado otros 17 posteriores a 2003, en su gran mayoría no hodgkinianos (LNH) (6-12).

Aunque la incidencia de estos LNH se ha incrementado en las últimas tres décadas y alrededor del $30 \%$ se manifiestan como una enfermedad extraganglionar, con frecuente afectación del hígado (en el 16-26\% de los casos en la biopsia y hasta un 56\% en la laparotomía), los LHP son muy raros, representando el $0,016 \%$ de todos los casos de LNH $(1,13,14)$. Un capítulo específico lo constituyen los procesos linfoproliferativos (exclusivamente hepáticos o extrahepáticos) tras el trasplante hepático, en cuyos receptores poseen una prevalencia del 2 al $4 \%$ en adultos y hasta el 20\% en niños (15-17).

Los LHP pueden presentarse a cualquier edad, si bien son más frecuentes en la quinta década, con una proporción hombre/mujer de 2 a 3 según las series $(1,5,10)$. Su patogenia no está definida, relacionándose con el virus de EpsteinBarr, virus C de hepatitis (VHC), virus de la inmunodeficiencia humana (VIH), cirrosis, lupus eritematoso sistémico y con el tratamiento inmunosupresor. Las situaciones de inmunodeficiencia en general y los virus juegan un papel significativo, en particular el de Epstein Barr 
(VEB) $(1,9,11,13,18-21)$. Este es un inductor de la proliferación policlonal de células $\mathrm{B}$, limitada por los linfocitos $\mathrm{T}$ en condiciones normales; al ser deficiente la función reguladora de las células $\mathrm{T}$ en pacientes inmunodeprimidos, las células B proliferan y pueden progresar a linfoma. Por su parte, el VHC es un virus linfotrópico que causa estimulación crónica de las células $\mathrm{B}$, su expansión policlonal y en ocasiones monoclonal. Nuestra paciente no había sufrido síntomas recientes de enfermedad infecciosa y la serología para esos virus fue negativa, lo que impide relacionar su LHP con ellos.

Los síntomas iniciales más comunes son dolor abdominal (39-70\%) y afectación del estado general, como ocurrió en el caso que comentamos, pudiendo cursar con síntomas B y excepcionalmente debutar con fallo hepático fulminante $(1,4,8)$.

En cuanto a las alteraciones bioquímicas, el perfil hepático se altera en el $70 \%$ de los casos; la LDH se eleva en un $30-80 \%$ de los pacientes y la $\beta_{2}$-microglobulina, marcador pronóstico, aumenta en el $90 \%$ de ellos $(1,5,18,22)$. Son menos comunes tanto una paraproteína monoclonal y la hipercalcemia, casi siempre mediada por calcitriol $(1,9,12,18,23)$.

En las técnicas de imagen, estos LHP pueden presentarse como masa única (39-60\% de los casos), lesiones múltiples (25-40\%) o como infiltración difusa $(1,18,21,25)$. En la ultrasonografía aparecen como áreas hipoecoicas o anecoicas y en la TC suelen manifestarse como lesiones hipodensas; tras la administración de contraste, un 50\% no lo captan, el $33 \%$ lo hacen de forma parcheada y un $16 \%$ lo hacen en anillo. En la resonancia magnética se describen como isointensas o hipointensas en $\mathrm{T} 1 \mathrm{e}$ hiperintensas en $\mathrm{T} 2$ $(1,2,5,10,21,22,24,25)$.

Histológicamente, los LHP pueden adoptar tres patrones: afectación difusa de los espacios portales (linfoma de bajo grado); infiltración difusa sinusoidal (linfoma de células T hepatoesplénico), o sustitución del parénquima por múltiples nódulos (linfoma de células grandes y alto grado de malignidad), como ocurrió en nuestro caso y con mucho el más frecuente. Asimismo, de los 121 casos descritos hasta 1997, en los 59 en los que se obtuvo el inmunofenotipo, 37 eran de células B, 15 de células T, y 7 de otros tipos $(1,19,21,22)$. Por otra parte, hasta 2005 sólo se habían descrito 16 linfomas del manto, generalmente asociados a cirrosis biliar primaria o infección por VHB y VHC $(1,26,27)$.

Como en otros tumores, indican un pronóstico desfavorable la edad avanzada, la afectación del estado general en el momento del diagnóstico, gran masa tumoral, subtipo histológico desfavorable, tasas elevadas de LDH y $\beta_{2}$ microglobulina, alto índice de proliferación, cirrosis y comorbilidad (1) .

En cuanto a su tratamiento, se ha sugerido que en LHP bien localizados y de pequeño tamaño, la cirugía, sola o combinada con quimioterapia, pudiera ser una opción a considerar $(1,5,22)$. Pero, dado que la recurrencia tras la exéresis no es infrecuente y que estos linfomas son quimiosensibles, es obligado incorporar la quimioterapia. El estudio realizado en el MD Anderson entre 1974 y 1995 , demostró remisión completa sólo con quimioterapia en el $83 \%$ de los casos y una supervivencia similar a los 5 años (17); unos resultados que, sin embargo, no fueron corroborados por otros grupos $(1,4,9)$.

Hasta 2001 la mediana de supervivencia en todos los pacientes con LHP era 15,3 meses, dependiendo del patrón de afectación hepática. Así, Emile y cols. observaron que en pacientes con patrón nodular la supervivencia a uno y tres años era del 70 y $57 \%$, respectivamente; pero, cuando predominaba la afectación difusa, la supervivencia caía al 38 y $18 \%$, respectivamente $(1,28)$.

La incorporación del rituximab a partir de 2002 en el tratamiento de los linfomas no hodgkinianos B ha incrementado de manera significativa las respuestas completas y la supervivencia de los pacientes tratados en la actualidad $(29,30)$. Ese anticuerpo quimérico monoclonal contra el antígeno CD20 de la superficie de las células B, ha significado una era nueva en este campo, hasta el punto de obligar a revisar los criterios del IPI previos a esa fecha (31). El rituximab hoy está siendo ensayado en procesos linfoproliferativos tras el trasplante hepático (32), es una opción como monoterapia de primera línea en el linfoma folicular y, junto con la terapia $\mathrm{CHOP}$, se recomienda en los LNH indolentes, intermedios y agresivos (29-34). Además, el desarrollo de sus derivados humanizados y radioinmunoconjugados, y su posible asociación con otros anticuerpos monoclonales, utilizados solos o combinados con CHOP, mejorará el pronóstico de los LNH refractarios a los tratamientos hoy disponibles.

En suma, y aunque infrecuente, el LHP debe ser incluido entre las causas de lesiones ocupantes de espacio en hígado. Con tal diagnóstico, nuestra paciente, que había sido ingresada con la sospecha de metástasis hepáticas múltiples de una neoplasia primaria no precisada y un pronóstico ominoso a corto plazo, tras tratamiento con R-CHOP, se encuentra en remisión completa y asintomática dos años después.

\section{BIBLIOGRAFÍA}

1. Noronha V, Shafi N, Obando JA, Kummar S. Primary non-Hodgkin's lymphoma of the liver. Crit Rev Oncol Hematol 2005; 53: 199207.

2. Levy AD. Malignant liver tumors. Clin Liver Dis 2002; 6: 147-64.

3. Oliva MR, Saini S. Liver cancer imaging: role of CT, MRI, US and PET. Cancer, Imag 2004; 4: S42-S46.

4. Caccamo D, Pervez NK y Marchewsky A. Primary lymphoma of the liver in acquired immunodeficiency syndrome. Arch Pathol Lab Med 1986; 110: 553-5.

5. Lei K. Primary non-Hodgkin's lymphoma of the liver. Leuk Lymphoma 1998; 9: 293-9.

6. Santos E, Raez L, Salvatierra J, Morgensztern D, et al. Primary hepatic non-Hodgkin's lymphomas: case report and review of the literature. Am J Gastroenterol 2003; 98: 2789-93.

7. Eom DW, Ryung J, Ku Kang Y, Sang Lee Y, et al. Clinicopathological features of eight Korean cases of primary hepatic lymphoma. Pathol Internatl 2004; 54: 830-6.

8. Cameron AM, Truty J, Truell J, Lassman C, et al. Fulminant hepatic failure from primary hepatic lymphoma: successful treatment with orthotopic liver transplantation and chemotherapy. Transplantation 2005; 15: 993-6. 
9. Hayashi H, Ohtani H, Ueda T, Yamaguchi J, et al. Primary hepatic lymphoma with spindle cell components: a case report. Virchows Arch 2006; 449: 591-6.

10. Salmon JS, Thomson MA, Arildsen RC, Greer JP. Non-Hodgkin lymphoma involving the liver: clinical and therapeutic considerations. Clin Lymphoma Myeloma 2006; 6: 273-80.

11. Nonami A, Takenaka K, Harada N, Kono K, et al. Primary hepatic lymphoma 1 year after resection of hepatocellular carcinoma. J Clin Oncol 2006; 24: 5784-6.

12. Gomyo H, Kagami Y, Kato H, Kawase T, et al. Primary hepatic follicular lymphoma: a case report and discussion of chemotherapy and favorable outcomes. J Clin Exp Hematopathol 2007; 47: 73-7.

13. Alexander DD, Mink PJ, Adami HO, Chang ET, et al. The nonHodgkin lymphomas: a review of the epidemiologic literature. Int J Cancer, 2007; 120(Supl. 12): 1-39.

14. Pulte D, Gondos A, Brenner H. Ongoing improvement in outcomes for patients diagnosed as having non-Hodgkin Lymphoma from the 1990s to the early 21 st century. Arch Intern Med 2008; 168: 469-76.

15. Domenech DE, de San José S, González-Barca E, Romagosa V, et al. Post-transplant lymphomas: a 20 year epidemiologic, clinical and pathologic study in a single center. Hematologica 2001; 86: 715-21.

16. Guthery SL, Heubi JE, Bucuvalas JC, Gross TG, et al. Determination of risk factors for EBV-associated post-transplant lymphoproliferative disorders in pediatric liver transplant recipients using objective case ascertainment. Transplantation 2003; 75: 987-93.

17. Avolio AW, Agnes S, Barbarino R, Magalini SC, et al. Post-transplant lymphoproliferative disorders after liver transplantation: analysis of early and end late cases ina 255 patient series. Transplant Proc 2007; 39: 1956-60.

18. Page RD, Romaguera JE, Osborne B, Medeiros LJ, et al. Primary hepatic lymphoma. Favorable outcome after combination chemotherapy. Cancer 2001; 92: 2023-9.

19. Rizzi EB, Schinina V, Cristofaro M, David V, et al. Non-Hodgkin's lymphoma of the liver in patients with AIDS: sonographic, CT, and MRI findings. J Clin Ultrasound 2001; 29: 125-9.

20. Baschinsky DY, Weidner N, Baker PB, Frankel WL. Primary hepatic anaplasic large-cell lymphoma of T-cell phenotype in acquired immunodeficiency syndrome: a report of an autopsy case and review of the literature. Am J Gastroenterol 2001; 96: 227-32.
21. Bronowicki JP, Bineau C, Feugier R, Hermine O, et al. Primary lymphoma of the liver: clinical-pathological features and relationship with HCV infection in French patients. Hepatology 2003; 37: 781-7.

22. Avlonitis VS, Linos D. Primary hepatic lymphoma: a review. Eur J Surg 1999; 165: 725-9.

23. Nan DN, Fernández-Ayala M, Terán E, Parra JA, et al. Severe hypercalcemia and solitary hepatic mass as initial manifestation of primary hepatic lymphoma. Liver 2001; 21: 159-60.

24. Rizzi EB, Schinina V, Cristofaro M, David V, et al. Non-Hodgkin's lymphoma of the liver in patients with AIDS: sonographic, CT, and MRI findings. J Clin Ultrasound 2001; 29: 125-9.

25. Maher MM, McDermott R, Fenlon HM, Conroy D, et al. Imaging of primary non-Hodgkin's lymphoma of the liver. Clin Radiol 2001; 56: 295-301.

26. Stancu M, Jones D, Vega F, Medeiros LJ. Peripheral T-cell lymphoma arising in the liver. Am J Clin Pathol 2002; 118: 574-81.

27. Gockel HR, Heidemann J, Lugering A, Mesters RM, et al. Stable remission alter administration of Rituximab in a patient with primary hepatic marginal zone B-cell lymphoma. Eur J Haematol 2005; 74: 445-7.

28. Emile JF, Azoulay D, Gornet JM. Primary non-Hodgkin's lymphomas of the liver with nodular and diffuse infiltration patterns have different prognoses. Ann Oncol 2001; 12: 1005-10.

29. Marcus R, Hagenbeek A. The therapeutic use of rituximab in nonHodgkin's lymphoma. Eur J Haematol Suppl 2007; 67: 5-14.

30. Fanale MA, Younes A. Monoclonal antibodies in the treatment of non-Hodgkin's lymphoma. Drugs 2007; 67: 333-50.

31. Sehn LH, Berry B, Chhanabhai M, Fitzgerald C, Gill K, et al. The revisited International Prognostic Index (R-IPI) is a better predictor of outcome than the standard IPI for patients with diffuse large B-cell lymphoma treated with R-CHOP. Blood 2007; 109: 1857-61.

32. González-Barca E, Domenech DE, Capote FJ, Gómez-Codina J, et al. Prospective phase II trial of extended treatment with rituximab in patients with B-cell post-transplant lymphoproliferative disease. Hematologica 2007; 92: 1489-94.

33. Coiffier B, Lepage E, Briere J. CHOP chemotherapy plus rituximab compared with CHOP alone in elderly patients with diffuse large B-cell lymphoma. N Engl J Med 2004; 346: 235-42.

34. Illidge T, Tolan S. Current treatment approaches for diffuse large B-cell lymphoma. Leuk Lymphoma 2008; 49: 663-76. 